\title{
Réflexions autour du vocabulaire grammatical dans le Dictionnaire de l'Académie francaise
}

\author{
Christophe Rey \\ Université de Picardie Jules Verne \\ Laboratoire d'Etudes Sociolinguistiques sur les Contacts de Langues et la Politique Linguistique (LESCLaP) \\ christophe.rey@u-picardie.fr
}

\section{Introduction}

Les travaux entrepris depuis le début des années 80 en matière d'informatisation des dictionnaires anciens offrent aujourd'hui des capacités et des facilités de travail considérables aux lexicographes et amoureux du lexique en tous genres. Notre communication se propose d'illustrer le type d'étude pouvant découler de la richesse offerte par les éditions électroniques des différents monuments de notre passé.

En nous appuyant sur la version informatisée du Dictionnaire de l'Académie Française [1], nous avons choisi de fournir une analyse diachronique d'un vocabulaire de spécialité : le vocabulaire grammatical [2].

Notre travail s'oriente autour de deux axes principaux : 1) un examen de l'évolution lexicale relative à ce champ particulier, et 2) une incursion au cœur des différents dispositifs de marquage du domaine grammatical à travers les éditions successives du dictionnaire des académiciens [3].

\section{De 1694 à 1932, histoire d'une maturation lexicale}

Deux raisons distinctes ont motivé le choix du Dictionnaire de l'Académie Française (désormais DAF) comme cadre de travail.

En premier lieu, si nous exceptons les parutions "clandestines" du Dictionnaire François de César-Pierre Richelet en 1680 (désormais $D F$ ) et du Dictionnaire Universel d'Antoine Furetière en 1690 (désormais $D U)$, le $D A F$ constitue le premier dictionnaire monolingue officiel dont l'état français s'est doté. Paru quelques années après le $D F$ et le $D U$, ce dernier constitue donc un témoin privilégié pour mener notre étude sur la théorisation du vocabulaire grammatical français.

D'autre part, la parution successive des diverses éditions du $D A F$ offre une opportunité toute trouvée de conduire l'étude diachronique que nous envisageons. Le parcours au sein de cet ouvrage va nous permettre de mettre en évidence les évolutions terminologiques relatives à un lexique de spécialité à travers l'une des vitrines institutionnelles françaises majeures.

\subsection{Constitution du corpus}

L'utilisation de la version électronique proposée sur CD-ROM par les éditions REDON nous a permis en quelques simples "clics" de pouvoir constituer notre corpus de travail. La collecte de ce dernier s'est faite en consultant les huit éditions simultanément et en appliquant un filtre sur le "domaine" de rattachement des articles. Nous avons ainsi relevé les différentes entrées lexicales étiquetées comme relevant du domaine de la Grammaire et introduites par des séquences du type "Terme de Grammaire", "Terme de grammaire grecque", "Terme de Grammaire Latine", etc. La Figure 1 ci-dessous fournit et schématise le décompte exact obtenu pour chaque édition : 


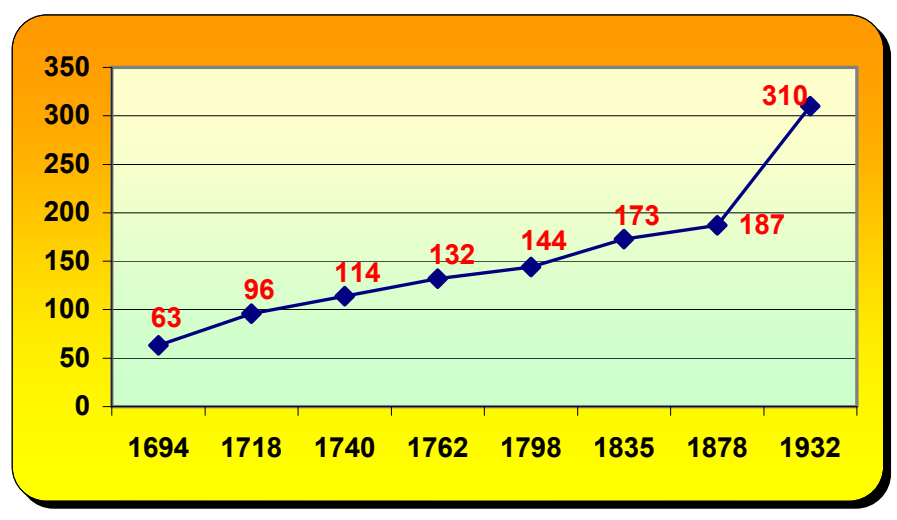

Figure 1. Répartition des articles grammaticaux dans les huit premières éditions du $D A F$.

La première constatation que nous faisons concerne la croissance régulière des articles relevant de ce vocabulaire de spécialité. Nous passons en effet d'un métalangage composé de 63 articles dans l'édition de 1694 à un métalangage composé de 310 articles dans celle de 1932. La proportion des entrées lexicales relevant du domaine grammatical a donc quasiment été multipliée par cinq entre la première et la huitième édition.

Soulignons également que cette courbe d'évolution possède une particularité intéressante en ce qui concerne l'édition de 1932. Cette dernière marque en effet un accroissement bien supérieur à celui constaté entre toutes les autres éditions puisqu'elle compte 123 entrées de plus que l'édition de 1878, alors que jusqu'à présent les éditions ne se différenciaient tout au plus que par une trentaine de lexies de l'une à l'autre.

La figure 2 fournit le détail de l'apport terminologique grammatical de chacune des éditions après 1694 [4]:

\begin{tabular}{|c|c|}
\hline ÉDITION & NOMBRE D'ARTICLES INTRODUITS \\
\hline $\mathbf{1 7 1 8}$ & 31 \\
\hline $\mathbf{1 7 4 0}$ & 17 \\
\hline $\mathbf{1 7 6 2}$ & 22 \\
\hline $\mathbf{1 7 9 8}$ & 10 \\
\hline $\mathbf{1 8 3 5}$ & 59 \\
\hline $\mathbf{1 8 7 8}$ & 13 \\
\hline $\mathbf{1 9 3 2}$ & 119 \\
\hline
\end{tabular}

Figure 2. Proportion d'articles nouveaux introduits dans les éditions du $D A F$ après 1694

Les données de notre tableau font état de quatre grandes phases dans l'évolution de la terminologie grammaticale du $D A F$, phases plus facilement identifiables sur le schéma suivant : 


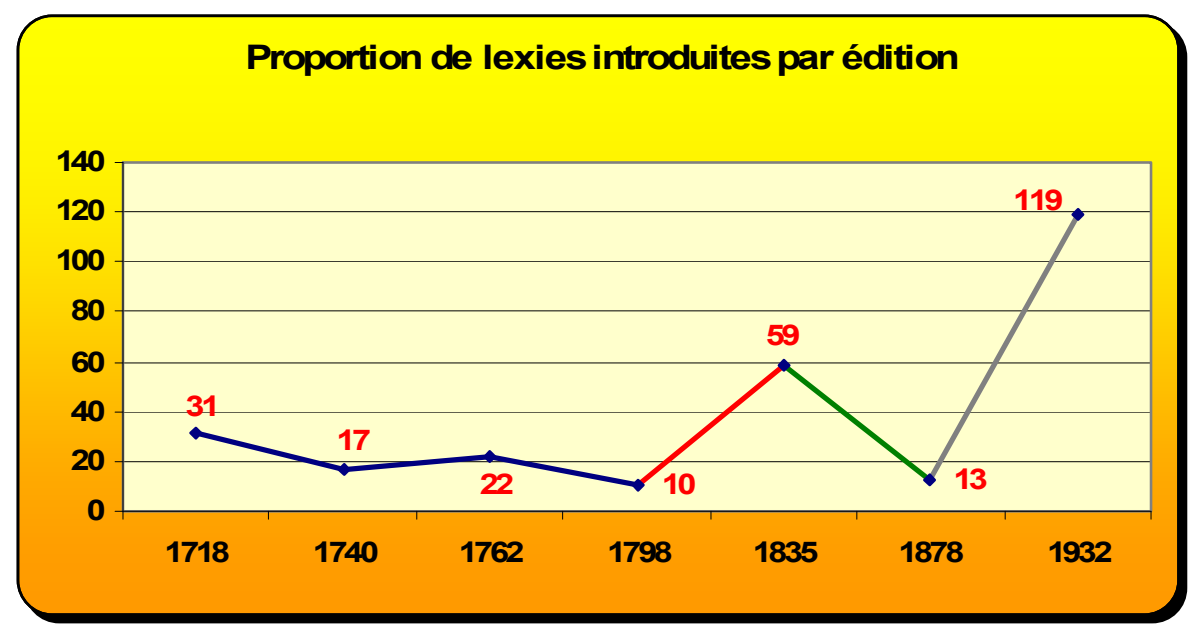

Figure 3. Les quatre grandes phases de l'évolution de la terminologie grammaticale du $D A F$

La première phase visible concerne les éditions de $1718,1740,1762$ et 1798 , pour lesquelles on peut constater un certain tassement des unités terminologiques introduites dans le vocabulaire grammatical. En effet, à la suite de l'édition publiée par l'abbé Régnier-Desmarais (1718) qui introduit 31 lexies de plus que celle de 1694, l'accroissement terminologique est constamment positif mais reste moindre. Même si l'édition de 1762 introduit 22 lexies, ces trois nouvelles versions du $D A F$ marquent un ralentissement dans l'accroissement $\mathrm{du}$ vocabulaire grammatical. Ceci semblerait montrer que la "mutation très forte $\mathrm{du}$ métalangage" qu'évoque J-C Chevalier (CHEVALIER 1998) et qui a éclaté "au milieu du XVIII ${ }^{\mathrm{e}}$ siècle dans les propositions de Girard (1747) et de Du Marsais dans l'Encyclopédie" (CHEVALIER 1998 : 304), ne s'est pas manifestée dans les éditions de 1762 et $1798 \mathrm{du} D A F$, tout au moins en ce qui concerne le vocabulaire grammatical.

L'Académie affirme pourtant explicitement, dans sa préface de 1762, vouloir prendre en considération ces avancées :

"Les sciences et les arts ayant été plus cultivés et plus répandus depuis un siècle qu'ils ne l'étoient auparavant, il est ordinaire d'écrire en François sur ces matières. En conséquence plusieurs termes qui leur sont propres, et qui n'étoient autrefois connus que d'un petit nombre de personnes, ont passé dans la Langue commune. Auroit-il été raisonnable de refuser place dans notre Dictionnaire à des mots qui sont aujourd'hui d'un usage presque général ? Nous avons donc cru devoir admettre dans cette nouvelle Edition, les termes élémentaires des sciences, des arts, et même ceux des métiers, qu'un homme de lettres est dans le cas de trouver dans des ouvrages où l'on ne traite pas expressément des matières auxquelles ces termes appartiennent." (DAF 1762, PRÉFACE)

Ce commentaire est une illustration du fait que "les mentalités et l'attitude varient" (BOUVEROT 1998 : 241), même chez les académiciens, mais il est dommage que cela se fasse encore "en fonction des spécialités" (BOUVEROT 1998 : 241) et que parmi ces spécialités ne figure pas la Grammaire.

Cette première phase contraste fortement avec le second mouvement qu'incarne l'édition de 1835. Cette édition traduit effectivement un renouveau terminologique du domaine grammatical, puisqu'elle n'introduit pas moins de cinquante-neuf nouveaux articles.

La troisième phase qu'illustre notre figure 3 est particulièrement intéressante puisqu'elle révèle une stagnation évidente de la maturation lexicale du champ grammatical. Avec l'édition de 1798 qui n'introduit que 10 articles nouveaux [5], l'édition de 1878, avec ses 13 articles introduits, est l'une des éditions les moins prolifiques. 
La quatrième et dernière phase décrite par notre tableau concerne l'accroissement massif proposé par l'édition de 1932. Dans cette huitième édition du $D A F$, cent dix-neuf articles de grammaire nouveaux sont introduits. Cette introduction massive contraste évidemment avec l'indigence de l'édition de 1878 et peut coïncider avec un nouvel élan de la lexicographie.

Peut-être pouvons-nous en effet voir à travers cet accroissement spectaculaire un héritage, non encore systématisé dans l'édition de 1878, de la parution du Grand dictionnaire universel du XIX siècle (18631876) de Pierre Larousse et du Dictionnaire de la langue française (1872) d'Émile Littré. L'une des preuves de ce que nous avançons est le nombre très important d'articles de Grammaire identifiés par Littré, chiffre qui se monte à cinq cent quatre-vingt trois articles.

La parution de l'ouvrage de Littré et de celui de Larousse a certainement poussé l'Académie à mettre à jour ses propres connaissances grammaticales.

\subsection{Analyse des évolutions}

Dans la seconde partie de ce premier chapitre nous souhaitons nous intéresser plus en détail aux évolutions constatées entre toutes les éditions. Nous allons pour cela étudier d'une part les articles unanimement recensés par toutes les éditions, avant de focaliser notre attention sur les articles propres à chacune des éditions.

\subsubsection{Les articles unanimement recensés}

Notre analyse diachronique permet de dégager un consensus de 44 articles recensés par toutes les éditions du $D A F$. Ces articles sont les suivants:

absolu, acception, accusatif, actif, adjectif, adversatif, aigu, antécédent, appellatif, apposition, augmentatif, auxiliaire, collectif, conjonctif, conjuguer, construction, construire, déclinable, déclinaison, décliner, défectif, dériver, disjonctif, formation, fréquentatif, gérondif, infinitif, inflexion, interrogatif, inversion, nombre, nominatif, oraison, participe, pluriel, possessif, prétérit, pronom, prosodie, quantité, régir, subjonctif, substantif, superlatif.

Le nombre de ces articles, qui constituent en quelque sorte le socle grammatical des huit premières éditions du $D A F$, revêt une importance qui varie en fonction des différentes éditions. Afin d'illustrer ces disparités proportionnelles, nous avons reproduit ci-dessous un tableau indiquant la part occupée par ce socle dans chaque édition :

\begin{tabular}{|l|l|l|l|l|l|l|l|l|}
\hline ÉDITION & 1694 & 1718 & 1740 & 1762 & 1798 & 1835 & 1878 & 1932 \\
\hline PROPORTION & $41 \%$ & $31 \%$ & $28 \%$ & $25 \%$ & $23 \%$ & $20 \%$ & $19 \%$ & $13 \%$ \\
\hline
\end{tabular}

Figure 4. Proportion des articles du socle grammatical dans les huit premières éditions du $D A F$

A la vue de ce tableau, il est clair que les 44 articles recensés par les huit premières éditions du DAF représentent, en relation avec l'accroissement progressif du vocabulaire grammatical souligné plus haut, un socle qui perd progressivement de son importance. Ne représentant plus que $13 \%$ de la totalité des articles grammaticaux de l'édition de 1932, la maigreur de ce socle illustre l'ampleur du renouvellement terminologique opéré au fil des siècles, et dont le point culminant figure dans les introductions massives de la huitième édition. 


\subsubsection{Termes propres à une édition}

Intéressons-nous à présent aux articles identifiés comme propres à une édition particulière. La figure 5 cidessous synthétise cette étude :

\begin{tabular}{|c|c|}
\hline ÉDITION & ARTICLES \\
\hline 1694 & Terme \\
\hline 1718 & $\varnothing$ \\
\hline 1740 & Adjectivement, Négative \\
\hline 1762 & Générique \\
\hline 1798 & Dichorée, Douteux [6] \\
\hline 1835 & Conclusif \\
\hline 1878 & Digamma, Permutable \\
\hline 1932 & $\begin{array}{l}\text { absolument, abstrait, accorder, activement, affixe, } \\
\text { allitération, ampliatif, analyse, antérieur, aphérèse, } \\
\text { apocope, apostrophe, appui, articulation, articuler, } \\
\text { aspiration, atone, atticisme, attraction, attribut, } \\
\text { attributif, attribution, bilabiale, bref, caractéristique, } \\
\text { casuel, causal, chuintement, chuinter, circonflexe, } \\
\text { circonstance, circonstanciel, commun, comparaison, } \\
\text { complément, composé, concession, concordance, } \\
\text { conditionnel, consécutif, conséquence, contracter, } \\
\text { contraction, coordination, coordonner, degré, } \\
\text { démonstratif, dénominatif, dépendance, déterminer, } \\
\text { diacritique, diminutif, direct, discours, dissylabique, } \\
\text { doux, explosif, figer, fini, forme, genre, gouverner, } \\
\text { homophone, homophonie, hybride, ictus, idiotisme, } \\
\text { imparisyllabe, incident, indirect, instrumental, } \\
\text { interrogation, intransitivement, invariable, juxtaposer, } \\
\text { labial, liaison, locution, logique, masculin, métathèse, } \\
\text { mouillure, moyen, négation, noble, objet, } \\
\text { onomastique, ordinal, oxyton, palatal, parisyllabe, } \\
\text { paronomase, paroxyton, particule, passé, péjoratif, } \\
\text { périodique, plus-que-parfait, principal, proparoxyton, } \\
\text { propérispomène, proposition, propre, provignement, } \\
\text { radical, séparable, simple, souscrire, subordination, } \\
\text { subordonner, suspensif, suspension, syncope, } \\
\text { synthétique, thématique, thème, tmèse, trisyllabe, } \\
\text { trisyllabique, variable. }\end{array}$ \\
\hline
\end{tabular}

Figure 5. Les articles propres à chaque édition.

Le tableau proposé fait apparaître un contraste frappant entre les sept premières éditions et l'édition de 1932.

En effet, alors que la huitième édition comporte 120 articles qui lui sont propres, les autres en comportent tout au plus 2. Dans le détail, nous constatons que les éditions de 1740, 1798 et 1878 introduisent deux articles qui ne seront pas repris ensuite, alors que les éditions de 1694, 1762 et 1835 n'en introduisent qu'un seul. L'édition de 1718 présente elle la particularité de n'introduire aucun article qui lui soit propre. 
Ces constatations ont le mérite de mettre en lumière une grande rigueur dans le travail d'introduction terminologique, ou tout au moins dans celui d'introduction des articles relatifs au domaine de la Grammaire.

Effectivement, si nous exceptons les articles introduits dans la huitième édition que nous n'avons pu analyser en fonction de la neuvième édition et qui ne sont donc pas significatifs, la proportion infime des articles propres à une édition particulière du $D A F$, autrement dit introduits dans une édition puis non repris par les éditions suivantes, témoigne d'une solide réflexion précédant la consécration lexicale des concepts grammaticaux manipulés. Cette prudence représente l'un des avantages majeurs du laborieux travail de définition des académiciens, souvent critiqué pour sa lenteur.

Ces mêmes constatations ont par ailleurs l'intérêt d'attirer notre attention sur certains articles propres à des éditions en particulier : les articles DICHORÉE et DIGAMMA notamment. Ces deux articles, respectivement marqués comme "Terme de Grammaire ancienne" et "Terme de Grammaire grecque" n'appartiennent donc pas à la grammaire française. Nous verrons dans la seconde partie de notre travail qu'ils ne sont pas les seuls mots issus de grammaires anciennes et pourtant répertoriés par le $D A F$, mais nous devons néanmoins nous attarder sur les raisons de leur présence.

Alors que DIGAMMA va disparaître dans les éditions suivantes, DICHORÉE sera repris dans les éditions de 1835 et 1878 en tant que "Terme de versification grecque et de versification latine", subissant ainsi un changement de marque de domaine. Aucun dictionnaire avant le $D A F$ ne mentionne le mot DICHORÉE comme un terme de Grammaire. Ce mot, ainsi que le mot DIGAMMA d'ailleurs, existe pourtant bien dans l'Encyclopédie de Diderot et d'Alembert mais n'est pas répertorié comme un terme de Grammaire. La justification de la présence de ces deux mots grammaticaux s'explique en fait - et nous revenons là sur ce que nous venons de dire - par leur emprunt au dictionnaire Grammaire \& Littérature (1782-1786) de Nicolas Beauzée et Jean-François Marmontel, issu de l'Encyclopédie Méthodique (1782-1832) de Charles-Joseph Panckoucke.

Dans ce dictionnaire, ces deux mots sont en effet répertoriés et appartiennent à la Grammaire. Ainsi que nous l'avons montré pour la terminologie relative à la description phonique de la langue [7], l'Encyclopédie Méthodique, à travers les théories de Beauzée a eu une incidence évidente, et notamment lexicale, sur les théories grammaticales de l'époque.

Afin d'achever cette première partie de notre travail nous souhaitons contraster l'existence de ces articles propres à une seule édition avec un certain nombre d'articles qui ont été adoptés au moins dans deux éditions avant d'être abandonnés par les éditions suivantes [8]. Ils sont répertoriés dans le tableau cidessous :

\begin{tabular}{|l|l|l|l|l|l|l|}
\hline 1694 & 1718 & 1740 & 1762 & 1798 & 1835 & 1878 \\
\hline & & & & adverbialité & adverbialité & adverbialité \\
\hline & alexandrin & alexandrin & alexandrin & & & \\
\hline anomal & anomal & anomal & anomal & anomal & & \\
\hline anomalie & anomalie & anomalie & anomalie & anomalie & & \\
\hline aoriste & aoriste & aoriste & aoriste & & & attiquement \\
\hline & & & & & & attiquement \\
\hline congru & congru & congru & congru & congru & & \\
\hline & & & & & figuratif & figuratif \\
\hline hétéroclite & hétéroclite & hétéroclite & hétéroclite & hétéroclite & hétéroclite & hétéroclite \\
\hline & & & hypallage & hypallage & hypallage & hypallage \\
\hline & & & & & imparisyllabique & imparisyllabique \\
\hline
\end{tabular}




\begin{tabular}{|c|c|c|c|c|c|c|}
\hline & & & & & incise & Incise \\
\hline & incongru & incongru & incongru & incongru & & \\
\hline & & & & & indéclinabilité & Indéclinabilité \\
\hline & & & & & interrogant & Interrogant \\
\hline \multirow[t]{3}{*}{ manger } & manger & manger & manger & manger & & \\
\hline & & & & & métaplasme & Métaplasme \\
\hline & & moeuf & moeuf & moeuf & moeuf & Moeuf \\
\hline \multirow[t]{12}{*}{ monosyllabe } & monosyllabe & monosyllabe & monosyllabe & monosyllabe & monosyllabe & Monosyllabe \\
\hline & & & nasal & nasal & & \\
\hline & & & & & nasalement & Nasalement \\
\hline & & & & & nasalité & Nasalité \\
\hline & & & négatif & négatif & négatif & Négatif \\
\hline & & & & & paulò-post-futur & paulò-post-futur \\
\hline & scander & scander & scander & scander & & \\
\hline & spondaique & spondaique & & & & \\
\hline & & & & Supplément & supplément & Supplément \\
\hline & & & synalèphe & synalèphe & synalèphe & Synalèphe \\
\hline & & & & & synchyse & Synchyse \\
\hline & voyelle & voyelle & voyelle & voyelle & voyelle & Voyelle \\
\hline
\end{tabular}

Figure 6. Les articles ayant disparu.

On peut se demander pourquoi ces articles ont été finalement abandonnés; nous n'analyserons pas ici les raisons de leur disparition, mais nous tenions toutefois à évoquer leur existence afin de fournir une illustration de la stratification du socle grammatical évoqué plus haut.

\section{Etude des marques de domaine grammatical}

Dans cette seconde partie de notre étude nous nous intéressons aux marques du domaine grammatical utilisées dans les différentes éditions du $D A F$.

Soulignons au préalable que le filtrage par domaine que propose l'édition électronique du $D A F$ offre une déperdition d'information non-négligeable importante à mentionner.

En effet, la sélection des articles en fonction de l'étiquette "Terme de Grammaire" nous renvoie pêle-mêle les articles possédant les marqueurs introductifs "Terme de Grammaire" (1694: AORISTE, AUGMENTATIF) mais aussi ceux possédant les marqueurs "En termes de Grammaire" (1694: AIGU, ANOMAL, AUXILIAIRE), "On dit en termes de Grammaire" (1694: CONGRU), et "On appelle en termes de Grammaire" (1718: RELATIF). Cette différence de marquage comporte un intérêt réel et a déjà fait l'objet de nombreux travaux [9]. De plus, ainsi que nous l'avons mentionné plus haut en note, ce filtrage automatique montre les limites de l'étiquetage appliqué puisque dans le type de requête que nous avons fait, certains articles comme DOUTEUX ( $D A F 1835$ et 1878) ou SUPPLÉMENT (DAF 1798) ne recevant que la mention "en Grammaire", ne sont pas identifiés comme appartenant au domaine grammatical. 
Dans cette communication, lorsque nous évoquons les articles marqués en "Terme de Grammaire", nous faisons allusion prioritairement à ceux possédant cette expression dans leur marque de domaine.

\subsection{Inventaire des marques de domaine grammatical}

Le tableau ci-dessous dresse l'inventaire des différentes marques du domaine grammatical utilisées pour les articles relevant de la Grammaire dans les huit premières éditions du $D A F$ :

\begin{tabular}{|c|c|c|c|c|c|c|c|c|}
\hline & $\mathbf{1 6 9 4}$ & $\mathbf{1 7 1 8}$ & $\mathbf{1 7 4 0}$ & $\mathbf{1 7 6 2}$ & $\mathbf{1 7 9 8}$ & $\mathbf{1 8 3 5}$ & $\mathbf{1 8 7 8}$ & $\mathbf{1 9 3}$ \\
\hline Terme de Grammaire & 62 & 94 & 112 & 127 & 141 & 163 & 175 & 293 \\
\hline Terme de Grammaire française & & & & 1 & & & & \\
\hline Terme de Grammaire latine & 1 & 2 & 2 & 3 & 2 & 1 & 2 & 4 \\
\hline $\begin{array}{c}\text { Terme de Grammaire grecque ou } \\
\text { latine }\end{array}$ & & & & 1 & & & & \\
\hline $\begin{array}{c}\text { Terme de Grammaire ancienne } \\
\text { Terme de Grammaire et de } \\
\text { musique }\end{array}$ & & & & & 1 & & & \\
\hline $\begin{array}{c}\text { Terme de grammaire grecque } \\
\text { Terme de logique et de } \\
\text { grammaire }\end{array}$ & & & & & & & 1 & 1 \\
\hline $\begin{array}{c}\text { Terme de grammaire et } \\
\text { d'administration }\end{array}$ & & & & & & & & 1 \\
\hline $\begin{array}{c}\text { Terme de grammaire hébraïque } \\
\text { TOTAL: }\end{array}$ & $\mathbf{6 3}$ & $\mathbf{9 6}$ & $\mathbf{1 1 4}$ & $\mathbf{1 3 2}$ & $\mathbf{1 4 4}$ & $\mathbf{1 7 3}$ & $\mathbf{1 8 7}$ & $\mathbf{3 1 0}$ \\
\hline
\end{tabular}

Figure 7. Les différentes marques du domaine grammatical recensées dans le $D A F$

\subsection{Nature des évolutions}

Le tableau obtenu fait apparaître plusieurs types d'évolutions relatives au marquage des articles appartenant au domaine de la Grammaire. Nous nous proposons de détailler ces évolutions.

\subsubsection{Evolution constante des articles marqués en "terme de Grammaire".}

L'une des premières conclusions qui se dégagent de cette étude concerne la présence majoritaire du marquage au moyen de la forme "Terme de Grammaire". Cette séquence représente en effet $98 \%$ des marquages dans les éditions de 1694, 1718, 1740 et 1798, 96\% dans l'édition de 1762, 94\% dans les éditions de 1835 et 1878 et enfin 95\% dans l'édition de 1932. Il s'agit là d'une domination sans partage avec les autres formes utilisées, et il est intéressant de constater que cette domination concerne chacune des éditions. Ceci tend à prouver que l'utilisation massive de cette forme coïncide avec l'accroissement du nombre d'articles de Grammaire à travers les éditions, traduisant ainsi un phénomène de cristallisation des 
connaissances grammaticales autour d'un patrimoine scientifique essentiellement français [10]. Ce phénomène contraste à première vue avec le phénomène de retour des grammaires anciennes que nous l'évoquerons plus bas, mais cette apparition simultanée d'étiquettes anciennes et modernes s'explique probablement par la conscience accrue des spécificités des langues qui marquent le XIX ${ }^{\mathrm{e}}$ siècle, loin de l'universalisme spontané des grammairiens du XVIII' siècle.

Ce phénomène s'apparente d'ailleurs étrangement au phénomène de "retour des grammaires anciennes" que nous évoquerons plus bas.

\subsubsection{Les nouvelles catégories}

Face à l'écrasante majorité des articles marqués en "Terme de Grammaire", figure un nombre très restreint de formes que nous avons choisi de regrouper sous la dénomination "catégories nouvelles" en raison de leur aspect plus marginal. Ces formes sont "Terme de Logique et de Grammaire" (1878 et 1932), "Terme de Grammaire et de Musique" (1835 et 1878), "Terme de Grammaire française" (1762) et "T. de Grammaire et d'Administration" (1932).

Ces formes méritent une attention particulière puisqu'elles systématisent toutes un travail original sur la catégorisation des articles grammaticaux.

Si nous considérons dans un premier temps ces formes dans leur ensemble, il semblerait, excepté la forme "Terme de Grammaire française", qu'elles illustrent la particularité lexicographique intéressante qui consiste à conserver au sein d'une même entrée, et d'une même définition d'ailleurs, des acceptions relatives à des domaines différents. Si nous tenons compte du fait que ces articles appartiennent tous aux éditions du $\mathrm{XIX}^{\mathrm{e}}$ ou du $\mathrm{XX}^{\mathrm{e}}$ siècle (voire aux deux), cette particularité contraste singulièrement avec la cristallisation évoquée plus haut et qui se traduit par un ancrage plus fort et individualisé de la Grammaire française dans le dictionnaire.

Si nous regardons à présent ces formes les unes après les autres, divers constats peuvent être dressés.

Tout d'abord, en ce qui concerne la forme "Terme de Grammaire française" qui caractérise le mot ALEXANDRIN dans l'édition de 1762, il semblerait que les académiciens, conformément à la définition qu'ils en donnent, aient voulu faire de ce mot un terme propre à notre Grammaire. Ceci est particulièrement ironique puisqu'il s'agit justement de la dernière édition où ce mot est considéré comme appartenant au domaine de la Grammaire.

La forme "Terme de Logique et de Grammaire" qui caractérise le mot PRÉDICAT est elle aussi intéressante puisqu'elle marque la prise en considération lexicale d'un terme déjà bien ancré dans les théories antérieures (Cf. PORT-ROYAL) et son adéquation aussi bien aux théories grammaticales qu'aux théories de la Logique.

Au-delà du fait de marquer l'appartenance du mot SYNCOPÉ à des domaines divers, la forme "Terme de Grammaire et de Musique" présente l'intérêt d'illustrer une nouvelle fois les imperfections et les carences de l'étiquetage des domaines proposé par la version électronique du $D A F$.

Notre tableau montre que la marque de domaine étudiée concerne les éditions de 1835 et 1878, toutefois, l'édition de 1798 semble avoir été lésée puisqu'elle marque également la double appartenance du mot aux domaines de la Musique et de la Grammaire, mais dégroupe l'acception grammaticale au sein d'une sousentrée.

Cette absence de marquage d'un point de vue informatique semble s'expliquer par le fait que l'appartenance du mot à la Grammaire est introduite par la forme "On dit en Grammaire". Comme nous l'avons dit plus haut, ce type de forme semble ne pas avoir été pris en compte lors de l'étiquetage des marques du domaine grammatical, et démontre encore l'imperfection de cette édition informatisée du $D A F$.

Pour finir, il nous semble que la forme "T. de Grammaire et d'Administration" qui qualifie le mot ONOMASTIQUE, en faisant référence au domaine de l'Administration uniquement mentionné à partir de 
l'édition de 1835, marque l'association d'un domaine ancien et en constante évolution (la Grammaire) avec un domaine moderne. La Grammaire, à travers son lexique et les interactions qu'elle occasionne avec des domaines plus récents, mûrit progressivement dans le $D A F$.

\subsubsection{Le retour des grammaires anciennes}

Un survol rapide des différentes marques de domaine grammatical utilisées par le $D A F$ suffit à illustrer ce que nous avons choisi de nommer le "retour des grammaires anciennes", à savoir un recours significatif à des termes issus des grammaires grecques, hébraïque ou latine.

Ce "retour" peut selon nous être justifié par deux mécanismes distincts que notre tableau met en évidence.

Le premier phénomène notoire qui nous permet de nous prononcer en faveur d'un rôle croissant des grammaires anciennes dans la Grammaire française est d'ordre chronologique.

Nous notons en effet que la publication des éditions successives du $D A F$ coïncide avec une cristallisation du recours à ce type de Grammaire. La forme "Terme de Grammaire Latine", présente dès l'édition de 1694 et dans toutes les éditions suivantes, montre que la seule influence avouée par les académiciens est celle du Latin.

A la suite de cette première référence ancienne, il faut attendre l'édition de 1762 pour constater un nouveau recours aux grammaires anciennes, et en l'occurrence une référence, à travers le mot SYNÉRÈSE, à la fois à la grammaire latine et à la grammaire grecque ("Terme de Grammaire grecque ou latine").

Malgré la disparition de la référence que nous venons d'évoquer dans l'édition de 1762, l'édition de 1798 introduit elle aussi une mention aux grammaires anciennes, en utilisant d'ailleurs une terminologie plutôt générique à travers l'expression "Terme de Grammaire ancienne".

Les éditions de 1835 et 1878 formulent quant à elles une référence à la grammaire grecque. Il s'agit là d'un tournant significatif dans le marquage des articles de Grammaire dans la mesure où le recours à la grammaire grecque concerne huit articles. Cette proportion est importante si nous tenons compte du fait que les mentions précédentes ne concernaient qu'un nombre bien plus restreint d'articles.

L'édition de 1932 est pour sa part encore plus significative car en plus de mentionner la grammaire grecque pour dix articles, elle fait une mention à la grammaire hébraïque. En faisant cohabiter des articles des grammaires latine, grecque et hébraïque, cette édition se distingue comme la plus tournée vers les grammaires anciennes. Ceci se vérifie également pour le second phénomène que nous comptons mettre en avant pour illustrer le "retour" des grammaires anciennes, à savoir le critère quantitatif.

Notre tableau montre qu'en plus d'introduire au fur et à mesure de ses éditions plus de termes des grammaires anciennes, le $D A F$ va avoir un recours quantitatif croissant à ces grammaires.

L'emprunt systématique de termes appartenant à la grammaire latine illustre effectivement ce phénomène puisque l'on passe de la mention d'un seul article dans l'édition de 1694 à celle de quatre articles dans l'édition de 1932.

Nous avons déjà mentionné les dix articles de cette huitième édition marqués comme appartenant à la grammaire grecque, mais insistons sur le fait que les éditions de 1835 et 1878 en mentionnent également huit chacune alors qu'aucune édition précédente - exceptée la mention à un terme de grammaire grecque ou latine dans l'édition de 1762 - ne cite cette grammaire.

En associant les quatre termes de grammaire latine, les dix termes de grammaire grecque et le terme de grammaire hébraïque de l'édition de 1932 on parvient à une édition qui compte dans son vocabulaire grammatical 15 articles constituant des termes de grammaires anciennes alors que l'édition de 1694 n'en compte qu'un.

Cette différence traduit bien évidemment une évolution des idées vis-à-vis des grammaires anciennes, entre une époque où le français venait de se doter d'un organisme scientifique d'État visant à faire du 
français une langue de prestige n'ayant plus à souffrir du joug du latin, et une époque où le comparatisme règne et où le prestige de la langue de Molière n'est plus à démontrer.

\section{Quelques réflexions autour de la microstructure}

Les réflexions livrées dans cette communication n'ont jusqu'ici porté que sur les aspects macrostructurels et non microstructurels du corpus étudié. Sans pour autant empiéter sur nos travaux en cours [11], nous souhaitons donc exposer à présent certaines différences microstructurelles repérées à travers notre corpus.

Ainsi que l'illustre l'accroissement progressif du nombre des articles explicitement rattachés au domaine de la Grammaire, le parcours diachronique auquel nous nous livrons offre un regard sur une maturation progressive de la science grammaticale. Depuis le siècle classique, ce type de connaissances ne cesse en effet d'être réexaminé et approfondi grâce à différentes avancées théoriques. La microstructure des 44 articles du corpus unanimement recensés par les huit premières éditions du $D A F$ se fait clairement l'écho de ces évolutions.

\subsection{Des définitions de plus en plus techniques}

L'examen microstructurel des articles de notre sous-corpus met en évidence un premier foyer d'évolutions significatives : la zone de définition. Au sein de la structure dictionnairique de notre sous-corpus, la définition fait en effet partie des éléments qui mettent le mieux en évidence l'évolution du discours grammatical à travers les huit premières éditions du $D A F$.

La définition peut par exemple être le lieu où se trouvent redistribuées les connaissances au sein du dictionnaire. Ainsi, pour l'article AIGU, alors que les sept premières éditions proposent en fait une définition de l'accent aigu :

"AIGU [...] En termes de Grammaire, On dit. Un accent aigu. C'est une petite marque qui se met sur une sillabe, pour montrer qu'elle doit estre prononcée d'un ton élevé." (DAF 1694, 1718, 1740, 1762, 1798, 1835, 1878)

la huitième édition ne définit plus ce type d'accent mais formule un renvoi vers une entrée plus adaptée à sa description : l'entrée ACCENT.

"AIGU [...] En termes de Grammaire, Accent aigu. Voyez Accent." (DAF 1932)

La définition peut également mettre en scène les progrès effectués au fil des siècles dans le domaine grammatical. Les articles ANTECEDENT, FORMATION, PARTICIPE, POSSESSIF, PRONOM et SUBJONCTIF illustrent parfaitement - et de manières distinctes - ce deuxième phénomène.

Chacun de ces articles traduit en effet une maturation établissant un contraste important entre les premières éditions du $D A F$ et celles proposées à partir du $\mathrm{XIX}^{\mathrm{e}}$ siècle. Plus précisément, ce sont les éditions de 1835 et 1932-35 qui semblent opérer les changements les plus remarquables.

L'édition de 1835 - édition ayant subi un important travail de refonte coïncidant visiblement avec une maturation des connaissances grammaticales - est ainsi le théâtre des changements relevés dans les articles PARTICIPE, POSSESSIF et SUBJONCTIF.

La sixième édition du $D A F$ opère un changement important dans l'article PARTICIPE, en requalifiant le participe en tant que "partie du discours" et non plus comme une "partie d'oraison" : 
"PARTICIPE. [...] Terme de Grammaire. Partie d'oraison." (DAF 1694, 1718, 1740, 1762)

"PARTICIPE. s. m. T. de Gram. Partie du discours qui est une des modifications du verbe." (DAF 1835)

Il est d'ailleurs intéressant de signaler que ce changement plus conforme à notre terminologie moderne est précédé d'une absence de classification dans l'édition de 1798 :

"PARTICIPE. sub. masc. Terme de Grammaire. C'est une modification du verbe." (DAF 1798)

puis d'une autre absence de classification dans l'édition de 1932-35 :

"PARTICIPE. n. m. T. de Grammaire. Forme verbale ainsi appelée parce qu'elle participe à la fois de la nature du verbe et de celle de l'adjectif."

L'article POSSESSIF est quant à lui le théâtre d'une première distinction entre les "pronoms possessifs" et les "adjectifs possessifs" :

"POSSESSIF. adj. m. T. de Gram. Il n'est usité que dans ces expressions, Pronom possessif, adjectif possessif, Pronom, adjectif qui sert à marquer la possession de la chose dont on parle. Mon, ton, son, nos, vos, leurs, sont des adjectifs possessifs." (DAF 1835, 1878, 1932-35)

Jusqu'à cette édition, les éditions précédentes ne faisaient en effet mention que des pronoms possessifs et proposaient même, ainsi que l'illustre les trois premières définitions, des exemples de ce que nous identifions aujourd'hui comme des adjectifs possessifs :

"POSSESSIF [...] Mon livre, vostre livre, ces pronoms mon \& vostre sont des pronoms possessifs. Mien, tien, sien, \&c. sont des pronoms possessifs relatifs.[...]" (DAF 1694, 1718, 1740)

Enfin, la définition de 1835 de l'article SUBJONCTIF offre la première apparition de la notion de "proposition subordonnée ou incidente" :

"SUBJONCTIF. s. m. T. de Gram. Mode du verbe, qui se place toujours après un autre verbe, ou une conjonction, et dans une phrase ou proposition subordonnée ou incidente. Le présent, l'imparfait, le plus-que-parfait du subjonctif. Que j'aime, que j'aimasse, que j'eusse aimé, sont au subjonctif $d u$ verbe Aimer. Les temps $d u$ subjonctif. Cette conjonction veut le subjonctif après elle. Édition 1835" (DAF 1835, $1878,1932-35)$

A l'image de l'édition de 1835, l'édition de 1932-35 du $D A F$ se distingue comme une édition également porteuse de changements majeurs au sein de la définition des termes grammaticaux. C'est notamment ce que nous constatons pour les articles ANTECEDENT, NOMBRE et PRONOM.

Se démarquant des éditions précédentes, l'article ANTECEDENT de la huitième édition mentionne la possibilité qu'un antécédent puisse être une proposition et non plus seulement un nom ou un pronom :

"Antecedent. s. m. En termes de Grammaire, Se dit des noms \& pronoms quand ils precedent \& regissent le Relatif qui," (DAF 1694, 1718, 1740, 1762, 1798, 1835, 1878)

"ANTECEDENT [...] En termes de Grammaire, il se dit d'un Nom, d'un pronom ou d'une proposition qui est déterminée par une proposition relative. Dans Dieu qui voit tout, Dieu est l'antécédent de la proposition relative qui voit tout." (DAF 1932-35) 
A l'article NOMBRE, les lexicographes de la huitième édition introduisent un complément d'information qui n'existe pas dans les éditions précédentes et qui stipule que la notion de "nombre" ne caractérise pas seulement les noms et les verbes, mais aussi les articles, les adjectifs et les pronoms :

"Nombre, Se dit en termes de Grammaire, des noms \& des verbes, selon qu'ils s'appliquent à une chose ou à plusieurs." (DAF 1718, 1740, 1762, 1798, 1835, 1878)

"Nombre se dit, en termes de Grammaire, des Noms, des articles, des adjectifs, des pronoms et des verbes, selon qu'ils s'appliquent à un seul être, à une seule chose ou à plusieurs. Nombre singulier. Nombre pluriel. Dans la plupart des langues, le nom et l'adjectif doivent s'accorder en genre et en nombre." (DAF 1932-35)

L'article PRONOM, quant à lui, illustre le même changement que celui mentionné plus haut à propos de l'article PARTICIPE de l'édition de 1835, à savoir une requalification du terme défini en "partie du discours" et non plus en "partie d'oraison".

"PRONOM. s. m. Terme de Grammaire. Celle des parties de l'Oraison, qui se met à la place du nom substantif." (DAF 1694, 1718, 1740, 1762, 1798)

"PRONOM. s. m. T. de Gram. Celle des parties d'oraison qui tient ou qui est censée tenir la place du nom substantif." (DAF 1835, 1878)

"PRONOM. n. m. T. de Grammaire. Celle des parties du discours qui tient ou qui est censée tenir la place du nom." (DAF 1932-35)

Les quelques exemples sur lesquels nous venons de nous attarder, nous permettent de montrer que la définition constitue l'un des champs dictionnairiques les plus propices aux changements microstructurels que connaissent les articles grammaticaux communs aux huit premières éditions du $D A F$.

\subsection{Une exemplification remaniée}

$\mathrm{Au}$ même titre que la définition, l'exemple - dont l'importance cruciale dans la microstructure dictionnairique n'est plus à démontrer [12] - semble lui aussi constituer un espace privilégié pour traduire l'évolution des connaissances au sein des 44 articles de notre sous-corpus. Plusieurs de ces derniers proposent en effet des disparités constatées au niveau de la zone d'exemplification, des disparités traduisant des évolutions particulièrement significatives.

En introduisant pour la première fois sous la forme d'exemples, les concepts de "dérivation" et de "composition", l'article FORMATION stigmatise ainsi une importante maturation dans la description morphologique de la langue française :

"FORMATION [...] Il désigne aussi, en termes de Grammaire, la Manière dont un mot se forme d'un autre mot, ou dont un mot passe par ses diverses formes. La formation du pluriel. La formation d'un temps, d'un mode. La formation de mots par dérivation, par composition, etc. Règles de formation." (DAF 1932-35)

Bien qu'introduite sous la forme d'exemples, la mention de l'opposition entre "dérivation" et "composition" marque incontestablement une rupture avec les articles des éditions précédentes, une rupture traduisant une plus grande maturité et une plus grande technicité des connaissances grammaticales de la huitième édition.

L'article DEFECTIF présente quant à lui la particularité de n'être doté qu'à partir de la huitième édition d'exemples servant à illustrer le concept de "verbes défectifs" :

"DÉFECTIF, IVE. adj. T. de Grammaire. Qui n'a pas tous ses temps et tous ses modes, en parlant des Verbes. Braire, frire, gésir sont des verbes défectifs." (DAF 1932-35)

L'absence d'exemples dans les sept premières éditions du dictionnaire constitue un manque évident que 
comble l'édition de 1932-35.

L'article NOMBRE déjà mentionné plus haut a la particularité d'illustrer le phénomène d'évolution des exemples au fil des éditions. En effet, alors que les cinq premières éditions du $D A F$ ne mentionnent comme exemples que les séquences "Nombre singulier. nombre pluriel", les trois éditions suivantes vont ajouter à celles-ci l'exemple suivant :

"NOMBRE [...] Dans la plupart des langues, le substantif et l'adjectif doivent s'accorder en genre et en nombre." (DAF 1835, 1878, 1932-35)

L'énoncé suivant, qui sonne comme une règle de Grammaire que l'on énonce solennellement, constitue un complément d'information bien utile.

Ce phénomène de multiplication des exemples au fil des éditions concerne également plusieurs autres articles, parmi lesquels figure l'article FREQUENTATIF. Ce dernier est en effet agrémenté de plusieurs illustrations supplémentaires au fil des éditions :

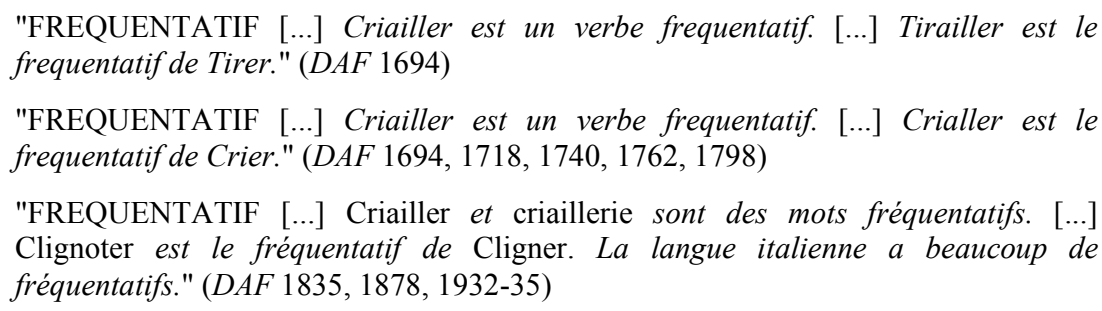

Les exemples d'articles de ce type pourraient aisément être multipliés parmi les 44 entrées retenues, aussi préférons-nous clôturer cette démonstration en insistant à nouveau sur le rôle crucial que joue la zone de l'exemplification dans l'évolution microstructurelle des articles de notre sous-corpus.

Le parcours microstructurel des articles de Grammaire communs aux huit premières éditions du $D A F$, mériterait sans aucun doute d'être étoffé par des développements autour de l'apparition dans certains articles de dégroupements polysémiques éventuellement accompagnés d'une numérotation des acceptions, ou alors par la mention de considérations d'ordre phonétique parfois formulées dans un nombre restreint d'articles. Nous réservons ces développements au travail de plus grande ampleur que nous menons par ailleurs et dont les conclusions sont encore à venir.

\section{Conclusion}

Cette petite excursion permise par l'outil informatique au cœur du métalangage grammatical des huit premières éditions du Dictionnaire de l'Académie française, est particulièrement riche d'enseignements.

Nous avons en effet pu voir s'affirmer progressivement une maturation et une structuration lexicale d'une discipline au fil des éditions, comme en témoignent à la fois le nombre croissant des entrées recensées, leurs évolutions microstructurelles et leur marquage systématique en tant que termes relevant du domaine grammatical français.

Le socle grammatical commun que nous avons pu mettre en évidence, ainsi que le nombre restreint des articles n'ayant qu'une durée de vie relative à une ou quelques éditions, illustrent par ailleurs la qualité du travail des académiciens dans la constitution de leur lexique grammatical. C'est certainement cette rigueur qui a fait de ce dictionnaire l'une des autorités lexicographiques les plus respectées sous laquelle se sont placés des ouvrages de grande notoriété comme ceux de Larousse, Robert, Bescherelle ou encore Littré.

Malgré les nombreuses critiques qui l'ont entouré et l'entourent encore, le $D A F$ apparaît comme un ouvrage rigoureusement structuré et dont l'intérêt pour la thématisation du vocabulaire grammatical est bien réel. 


\section{Notes}

[1] Les éditions Champion n'ayant pas encore proposé de version électronique des éditions successives du Dictionnaire de l'Académie Française, nous avons du avoir recours aux produits de la maison d'édition REDON.

[2] Notons que nous avons fait paraître récemment (Rey 2007) une étude similaire, portant sur le vocabulaire grammatical présent dans le Dictionnaire françois (1680) de César-Pierre Richelet.

[3] En raison du caractère incomplet de la neuvième édition du $D A F$ sous sa forme électronique, notre étude ne porte que sur les huit premières éditions de ce dernier.

[4] Ces chiffres ne tiennent pas compte des articles qui disparaissent d'une édition à l'autre.

[5] La pauvreté de l'édition de 1798 est étonnante dans la mesure où cette édition est généralement reconnue pour marquer une introduction massive des lexiques de spécialité.

[6] L'exemple de l'article DOUTEUX montre une faiblesse de l'étiquetage effectué sur les marques de domaine. Ce mot est introduit par la séquence "En Grammaire" dans les éditions de 1835 et 1878 mais ne figure pas dans la liste des termes appartenant au domaine grammatical.

[7] Cf. REY (2004).

[8] En tant que termes appartenant au domaine de la Grammaire.

[9] Cf. notamment GLATIGNY (1984) et BRANCA/REY (2004).

[10] Nous admettons ici provisoirement que la simple mention "Terme de Grammaire" est un renvoi à la Grammaire française.

[11] Nous effectuons actuellement une analyse fine des évolutions microstructurelles des articles de Grammaire du $D A F$, mais nos conclusions ne sont à ce jour encore que trop parcellaires pour être exposées ici.

[12] Cf. "L'exemple lexicographique dans les dictionnaires français contemporains", Actes des "Premières Journées allemandes des dictionnaires", ed. Michaela Heinz, Niemeyer, Coll Lexicographica, Series Maior, 2005.

\section{Références bibliographiques}

Sources primaires

Beauzée, N, Marmontel, J-F. (1782-1784-1786). Encyclopédie Méthodique. Grammaire \& Littérature. A Paris (chez Panckoucke), Liège (chez Plomteux). 3 vol.

Encyclopédie méthodique ou par ordre de matières par une société de gens de lettres, de savants et d'artistes ; précédée d'un Vocabulaire universel, servant de Table pour tout l'Ouvrage, ornée des Portraits de MM. Diderot et d'Alembert, premiers Editeurs de l'Encyclopédie. (1782-1832). A Paris (chez Panckoucke), Liège (chez Plomteux). 210 vol.

Encyclopédie de Diderot et d'Alembert ou Dictionnaire raisonné des sciences, des arts et des métiers, cd-rom pc, version 1.01, Redon, 1999.

Furetière, A. (1690). Dictionnaire Universel, Dictionnaires des XVI ${ }^{\mathrm{e}}$ et XVII ${ }^{\mathrm{e}}$ siècles, 1998, cd-rom pc, version 1.0, Champion électronique.

Larousse, P. (1863-1876). Grand dictionnaire universel du XIX ${ }^{\mathrm{e}}$ siècle [CD-ROM]. Genève : Ed. Champion électronique, 2002.

Le Dictionnaire de l'Académie française : histoire et nuances de la langue française (1694-1935). (2000). éditions Redon. 
Littré, E. (1872). Dictionnaire de la langue française [CD-ROM], Le grand atelier historique de la langue française, 2001.

Richelet, C-P. (1680). Dictionnaire françois, Dictionnaires des XVI et XVII ${ }^{\mathrm{e}}$ siècles, 1998, cd-rom pc, version 1.0, Champion électronique.

Sources secondaires

Bouverot, D. (1998). Mots nouveaux et vocabulaires spéciaux dans le Dictionnaire de l'Académie, Actes du Colloque international Le Dictionnaire de l'Académie française et la lexicographie institutionnelle européenne, Institut de France, novembre 1994; (ed. B. Quemada \& J. Pruvost), Paris, Champion : 237-252.

Branca, S., Rey, C. (2004). Note sur le traitement des entrées grammaticales communes aux trois dictionnaires français monolingues du XVII ${ }^{\mathrm{e}}$ siècle, Cahiers de Lexicologie n 84, pp. 23-41.

Chevalier, J-C. (1998). Le Dictionnaire de l'Académie française et la grammaire, Actes du Colloque international Le Dictionnaire de l'Académie française et la lexicographie institutionnelle européenne, Institut de France, novembre 1994; (ed. B. Quemada \& J. Pruvost), Paris, Champion : 295-307.

Glatigny, M. (1984). Polysémie et homonymie dans le Thresor de Nicot, Matériaux pour une histoire des théories linguistiques, p. 249-258. Lille, P.U.L.

Heinz, M. (ed) (2005). L'exemple lexicographique dans les dictionnaires français contemporains, Actes des "Premières Journées allemandes des dictionnaires", Niemeyer, Coll Lexicographica, Series Maior.

Rey, C. (2007). La Grammaire dans le Dictionnaire françois (1680) de César-Pierre Richelet, L'Information grammaticale, 114, pp. 20-24.

Rey, C. (2004). Analyse et informatisation des articles traitant de l'étude des sons dans le dictionnaire Grammaire \& Littérature de Nicolas Beauzée et Jean-François Marmontel, issu de l'Encyclopédie Méthodique. Thèse de doctorat. Aix-en-Provence. 